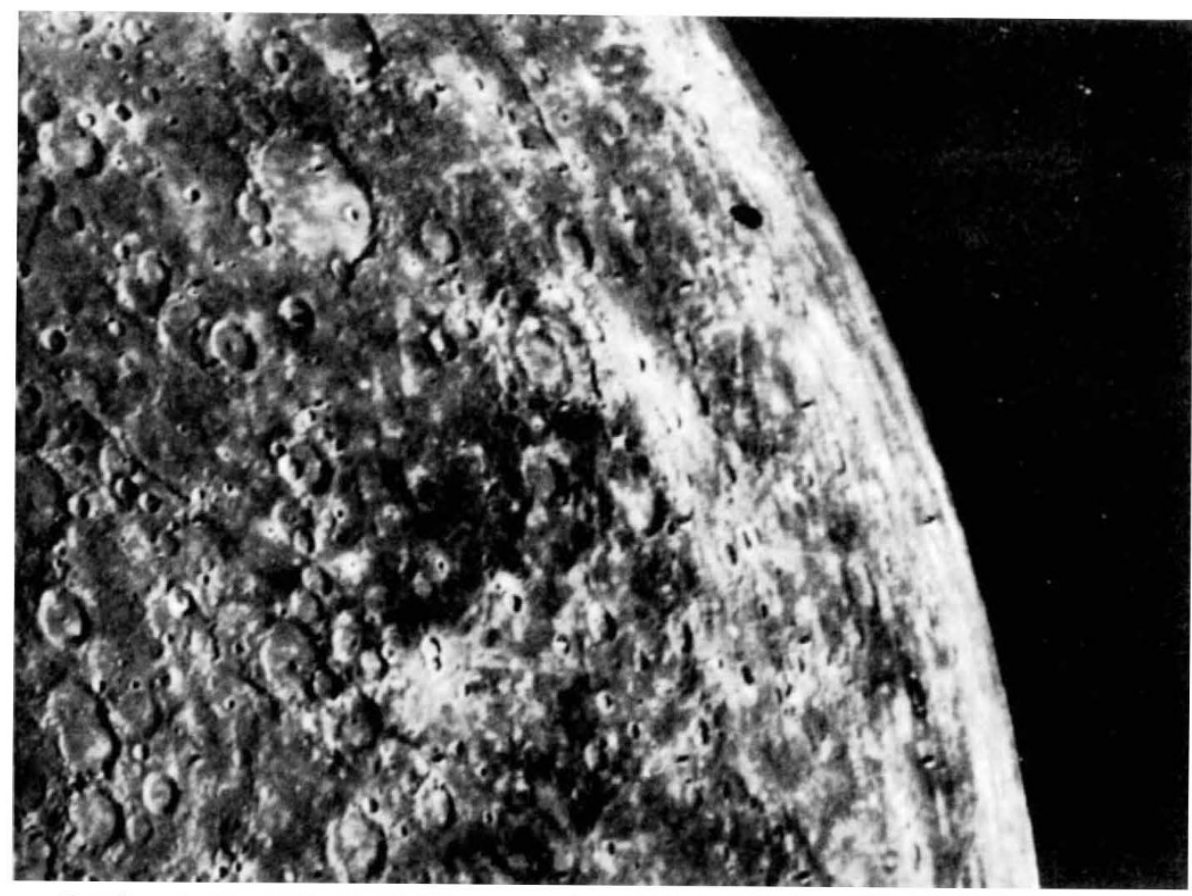

Southwestern quadrant of Mercury, viewed from Mariner $104 \mathrm{~h}$ before closest approach. Distance of spacecraft from planet was $198,000 \mathrm{~km}$; the largest craters visible are some $100 \mathrm{~km}$ across. (NASA photograph.)

atmosphere could be seen have been made by members of that fraternity from time to time, but met with a cool reception from the professionals. Wise after the event, those professionals now say that such a tenuous atmosphere can be maintained, in spite of the low escape velocity from the planet, because of particles released through radioactive decay. Uranium releases helium (alpha particles), and potassium yields argon; another effect, capture from the solar wind, probably accounts for the presence of neon.

Clearly it will take a while for the new information to be digested and incorporated into theoretical models, and in September there is the prospect of more information as Mariner 10 passes by Mercury again. In the meantime, it is interesting to reflect on a comment attributed to Bruce Murray, leader of the Mariner $10 \mathrm{TV}$ experiment team (Aviat. Week Space Technol., 100 (14), $16 ; 1974)$, which indicates the rapid development of spacecraft systems and also answers the 'doubts' of that radio astronomer. The Mariner 10 pictures cannot be directly compared with Lunar Orbiter pictures, it seems, "because the Mariner 10 photos show more detail".

\section{Models of growth and competition}

from our Animal Ecology Correspondent THE dynamics of competitive interaction between two species have long been the target of approximations. The formula

$$
\frac{d N_{i}}{d t}=r_{i} N_{i}\left(1-N_{i} / K_{i}-\alpha_{i j} N_{j} K_{i}\right)
$$

(where $N_{\mathrm{i}}$ is the density of species $i$; $r_{i}$ is the exponential growth rate of this species when its population density and that of its competitor $j$ are low; $K_{i}$ is the carrying capacity of the environment for species $i$ when its competitor $j$ is absent; $\alpha_{i j}$ is the linear reduction (of $K_{i}$ ) of species $i$ 's growth rate caused by its competitor $j$ ) has stood for more than 50 years since being proposed by Lotka (Elements of Physical Biology, Williams and Wilkins, Baltimore, 1924) and Volterra (Leçons sur la Theorie Mathematique de la Lutte pour la Vie, Gauthier-Villars, Paris, 1931). The model was validated in 1934 by Gause (The Struggle for Existence, Williams and Wilkins, Baltimore) who studied the dynamics of yeast and protozoan competition. The linearity of the model suited this kind of interaction. For arthropod interactions the model is less perfect. Gilpin and Ayala (Proc. natn. Acad. Sci. U.S.A., 70, 3590; 1973) have recently put the model to the test using arthropod species and have come up with some important modifications.

Their competing species were Drosophila willistoni and $D$. pseudoobscura. Adult flies, in known densities, were put in culture vessels containing known amounts of food. After 1 week the surviving adults were counted, as were their offspring which emerged from the culture medium during the following 4 weeks. Zero isoclines, which depict the separation point between positive and negative population growth, were constructed from continuous populations, maintained by serial transfer. The single species equilibrium, or environmental carrying capacity, is the maximum population the culture medium can stand. As a competitor increases in abundance the zero isocline falls away from the maximum point. Where it intersects the zero isocline for the competitor species is the equilibrium point for the interaction of the two species.

The data from the Drosophila cultures were fitted to a large number of competition models chosen according to the criteria of simplicity, reality, generality and accuracy. The model that stood up best was

$$
\left.\frac{d N_{i}}{d t}=r_{i} N_{i}\left(1-N_{i} / K_{i}\right)^{\theta^{i}-\alpha_{i j}} N_{j} K_{i}\right)
$$

The interpretation of the parameters is straightforward. $r_{i}$ is the exponential rate of growth of species $i$ when its density and that of species $j$ is low; $K_{i}$ is the carrying capacity of the environment for species $i$ in the absence of its competitor $j ; \alpha_{i j}$ is the reduction of growth rate of $i$ as a result of the activities of $j$, and $\theta i$ denotes the asymmetry of the single species growth rate of species $i$. In the absence of competition, that is when $x=0$, the growth rate conforms to the logistic. In other words the relationship between growth rate and density is parabolic. The point of maximum growth rate is $K / 2$. The experimental data pointed out this restriction. The addition of $\theta$ removes it and allows the point of maximum growth rate to be less than, or more than, $K / 2$.

This is an important modification to the Lotka-Volterra equation. Vertebrate populations in which growth rate tends to be controlled abruptly by the imposition of territoriality and other social factors cannot adequately conform to the continuously linear fashion demanded by the old model (when $\theta=1$ ). For them $\theta$ will tend to be larger than 1. Invertebrate populations, in which eggs, larvae and pupae are ignored, will tend to have a $\theta$ value less than 1 .

Gilpin and Ayala propose this new model to encompass intraspecific and interspecific competition. Population ecologists shall have to wait before assessing its general applicability.

\section{Biogenesis of chloroplasts and mitochondria}

\section{from a Correspondent}

RECENT work on the biogenesis of chloroplasts and mitochondria was discussed at a symposium of the British Society for Cell Biology at the University of Warwick on March 28-29. Both 